\title{
Desigual dades na distribuição da cárie dentária em adolescentes de Indaiatuba (SP), 2004
}

\author{
Inequalities in distribution of dental caries \\ in teenagers of Indaiatuba, São Paulo State, 2004
}

Lilian Berta Rihs ${ }^{1}$

M aria da Luz Rosário de Sousa ${ }^{1}$

Silvia Cypriano ${ }^{2}$

Nádja M oscoso Abdalla ${ }^{3}$

${ }^{1}$ Departamento de

Odontologia Social,

Universidade Estadual de

Campinas. Av. Limeira 901,

Areião. 13414-903

Piracicaba SP.

lilianberta@yahoo.com.br

${ }^{2}$ Centro de Ciências da

Vida, Faculdadede

Odontologia, Pontifícia

Universidade Católica de

Campinas.

${ }^{3}$ Departamento Regional de SaúdeXII.
Abstract The objectives of this work was to know the dental caries prevalence and treatment need, besides the distribution of the dental caries in a population of 12 year-old teenagers with high incidence of dental caries. 309 adolescents of public schools of Indaiatuba, São Paulo State, in 2004, were examined. They were selected by process systematic random and theexamsfollowed theWorld Health Organization criteria. The percentile of school cariesfreewas of $38.8 \%$. Among theadolescents examined, $36.6 \%(n=113)$ presented dental caries. DM FT was of 2.50 (IC $95 \%=2.07-2.93)$. The decayed component was the highest $(61.0 \%)$. The coefficient of Gini was 0.602 and $\mathrm{SiC}$ value was of $5.97($ IC $95 \%=5.32-6.62)$; in this group especially, $74.7 \%(n=74)$ presented dental caries activity in the moment of the exam. Thus, it can be concluded that the adolescents of this city presented low dental caries experience; however, their needs were not assisted, oncemost of DM FT wasformed of the decayed component at 12 years old. In the group $\mathrm{SiC}$, it was verified that this came quite high, being recommen ded actions addressed to this specific group of adolescents.

Key words Oral health, Epidemiology, Dental caries
Resumo Os objetivos deste trabalho foram conhecer a prevalência da cárie dentária ea necessidade de tratamento odontológico, além da distribuição da cárie dentária na população que apresenta índices de cárie mais elevados em escolares dedozeanos. Foram examinados 309 adolescentes, de escolas públicas de Indaiatuba (SP), em 2004, selecionados mediante processo amostral aleatório sistemático e os exames seguiram os critérios da Organização M undial de Saúde. 0 percentual de escolares livres de cárie foi de 38,8\%. Dentre os escolares examinados, 36,6\% ( $n=113$ ) apresentaram atividade de cárie. O CPOD foi de 2,50 (IC95\% $=2,07-2,93)$. 0 componente cariado foi 0 mais prevalente $(61,0 \%)$. 0 coeficiente de Gini foi 0,602 e o valor do índice SiC foi de 5,97 (IC95\%=5,32-6,62); neste grupo em especial, $74,7 \%(n=74)$ apresentaram atividade de cárieno momento do exame. Desta forma, pode seconcluir que os escolares deste município apresentaram baixa experiência de cárie; entretanto, não apresentaram suasnecessidadesatendidas, uma vez que a maior parte do CPOD era formada do componente cariado, aos doze anos de idade. No grupo $\mathrm{SiC}$, verificou-se que este apresentou-se bastante elevado, sendo recomendadas ações direcionadas a este grupo específico de adolescentes.

Palavras-chave Saúde bucal, Epidemiologia, Cárie dentária 
Introdução

$\mathrm{N}$ as últimas décadas, tem sido verificado um declínio tanto da prevalência quanto da severidade da cárie dentária em várias localidades em crianças aos doze anos de idade, o que vem sendo atribuído principalmente ao aumento da exposição a fluoretos ${ }^{1-3}$.

No Estado de São Paulo, alguns trabalhos também vêm apontando esta redução na experiência de cáries aos doze anos de idade ${ }^{1,4,5}$, atingindo inclusive as metas da Organização M undial de Saúde(OM S) para o ano 2000 para a idade índice de doze anos. Entretanto, em al guns trabalhos que verificam as desigualdades na distribuição da cárie, utilizando instrumentos como $\mathrm{SiC}$ (Significant CariesIndex) ecoeficientedeGini, dentre outros, nota-se que estas desigualdades têm aumentado, ou seja, apesar da diminuição na experiência de cárie na população como um todo, algumas minorias ainda apresentam índices elevados de cárie dentária ${ }^{6-10}$. Dados de um estudo ecológico realizado no Estado deSão PauIo no ano de 1998 mostram associação entre piores condições socioeconômicas dos municípios (SiC, Coeficiente de Gini, proporção de crianças livres de cárieeíndice de desenvolvimento humano - IDH) edistribuição de cárie em crianças de onze e doze anos de idade. Em geral, crianças de municípios com mel hores condições de vida apresentavam menor prevalência de cáriee, simultaneamente, maior desigualdadena distribuição da cárie dentária 6 .

Assim sendo, os objetivos deste trabal ho foram conhecer a prevalência da cárie dentária e necessidade de tratamento odontológico, identificar os aspectos epidemiológicos na distribuição da cárie dentária na população que apresenta índices de cárie mais elevados em adolescentes dedoze anos deidade do município del ndaiatuba, no ano de 2004.

\section{Métodos}

O município de Indaiatuba está local izado a 112 quilômetros de São Paulo e possui cerca de 160.000 habitantes. As águas de abastecimento público são fluoretadas desde 1979, com aproximadamente $94,4 \%$ das residências favorecidas com abastecimento de água. $\mathrm{O}$ IDH (índice de desenvolvimento humano) no município foi de 0,83 , em 2000 , sendo maior que a média geral do estado para o mesmo ano, que foi de 0,81 .
Para o cálculo do tamanho da amostra, utilizou-se o valor da média (CPOD foi de 1,84 aos doze anos) e desvio padrão $(\mathrm{dp}=2,13)$ obtidos em um estudo realizado no ano de 2000 , adotando-se para tal nível de confiança de $95 \%$, precisão de $20 \%$ e efeito do desenho (deff) de2. Somaram-se $20 \%$ a este total, para compensar eventuais perdas e recusas, resultando em um tamanho amostral de 309. A amostra foi probabilística, adotando-se a regra de não reposição dos elementos amostrais.

O sorteio dasinstituições deensino teve como base a relação das escolas municipais e estaduais fornecidas pela Secretaria de Educação, frequentadas por indivíduos de doze anos. As escolas especializadas em crianças portadoras de necessidades especiais foram excluídas do sorteio. Procedeu-seao sorteio ponderado, de tal forma que as escolas com maior número de alunos tiveram mais chance de serem sorteadas, seguindo as recomendações adotadas no Levantamento N acional $^{11}$. O sorteio dos elementos amostrais também seguiu estas recomendações.

Esta pesquisa se iniciou após a aprovação do Comitê de Ética em Pesquisa da Faculdade de Odontologia de Piracicaba. A seguir, através do termo de consentimento livree esclarecido, obteve-se a autorização dos pais ou responsáveis para que os escolares pudessem participar do presente estudo.

Os códigos e critérios utilizados para a determinação do índice CPOD, que medea experiência de cárie dentária, assim como os critérios para indicação das necessidades detratamento, seguiram as atuais recomendações da Organização M undial deSaúde $(\mathrm{OMS})^{12}$. Ressalta-sequetambém trabal hamos com resultados secundários referentes aos anos de 1992, 1993 e 1994; estes levantamentos seguiram as recomendações propostas pela OM S, na sua terceira edição ${ }^{13}$. Desta forma, a comparação é feita apenas a título de ilustração de uma tendência de declínio na experiência de cárie no município enenhum testeestatístico foi realizado com os dados referentes a estes anos.

Para a indicação de selantes, adotaram-se as regras de decisão sugerida pela SES/SP ${ }^{14}$, sendo 0 selante indicado apenas na presença simultânea das seguintes condições: dente presente há menos de dois anos, presença de cárieou história de cárieno dentehomólogo epresença de placa bacteriana clinicamente detectável, evidenciando higiene bucal precária.

Em adição, os escolares foram avaliados quanto à presença de atividade de cárie, tendo como 
base alguns sinais clínicos definidos por NYVAD et al. ${ }^{15}$. A pós um cuidadoso exame em todos os dentes do indivíduo para investigar sinais de atividade de cárie segundo a reflexão e textura da superfície dentária (ativa ou inativa), os indivíduosforam incluídosem dois grupos: indivíduo sem atividade de cárie (código 0) e indivíduo com atividade de cárie (código 1). N esta categoria, foram incluídososindivíduosem quese observou a presença de lesões em esmalte com sinais de atividade de cárie, como superfície com aspecto de giz, opaca e rugosa, associada à presença de placa bacteriana e/ou gengivite marginal e/ou presença de lesão de cárie aguda em dentina (tecido com aspecto claro e com descamação).

Para a análise dos resultados, utilizou-se 0 Coeficiente de Gini, para a verificação das desigualdades na experiência de cárie nesta população ${ }^{16}$. Além disto utilizou-se o Significant Caries Index - SiC Index, calculado separando-se do grupo geral um terço dos indivíduos que tenham apresentado os valores mais altos de experiência de cárie. N este subgrupo, calcula-se a média CPOD, sendo este o valor do SiC. Para a realização de al gumas análises neste trabal ho, o SiC foi comparado com o restante da população, ou seja, um terço da população compôs o SiC e os outros dois terços foram compostos pelo restante da população que não se enquadrou nos crité rios para a definição do $\mathrm{SiC}$, podendo, ainda, ser considerados como os dois terços da população que apresentaram os menores índices de cárie.

A calibração da equipe, formada por dois cirurgiões-dentistas, foi realizada em 36 horas, divididas entre discussões teóricas e atividades práticas, simulando as diferentes condições esituações que os profissionais encontrariam durantea realização do trabalho prático. A porcentagem deconcordância interexaminadores para cáriefoi 96\% e de $95 \%$ para as necessidades de tratamento.

Durante a realização dos exames, aferiu-se em cerca de $10 \%$ da amostra examinada a percentagem de concordância intra-examinadoras, obtendo-se um val or acima de $90 \%$ para cárie enecessidades de tratamento, estando desta forma dentro dos limites aceitáveis e recomendados pela $\mathrm{OM} \mathrm{S}^{13}$.

Os exames foram realizados em local com luz natural, utilizando-se espelho bucal plano e sonda CPI para levantamentos epidemiológi$\cos ^{12}$. Os dados foram registrados por anotadores previamentetreinados, em fichas individuais, cujo modelo simplificado foi proposto pela OM S com adaptações específicas para esta pesquisa.

A análise dos resultados foi realizada através do softwareEpil nfo. Para a comparação entre as médias CPOD entre os anos de 2000 e 2004, foi usado o teste $M$ ann-Whitney. Para a realização do cálculo do Coeficiente de Gini e a confecção da curva de Lorenz, utilizou-seum planilha específica para este fim, em Excel ${ }^{16}$.

\section{Resultados}

Foram examinados 309 escolares de doze anos de idade matriculados em escolas públicas do município del ndaiatuba (SP), em 2004. A amostra foi composta de 141 pessoas do sexo masculino (45,6\%) e 168 do sexo feminino (54,4\%).

Em 2004, 38,8\% ( $n=120$ ) dos escolares examinados apresentaram-selivrede cáries ( CPOD $=0$ ). Observou-se que $36,6 \% \quad(n=113)$ dos escolares examinados apresentaram atividade de cárie.

A média CPOD aos doze anos foi de 2,50 (IC95\% $=2,07-2,93)$, sem diferença segundo 0 sexo $(p=0,702)$. Entreosindivíduos com experiência de cárie (CPOD>0), O CPOD foi de 4,1 (IC 95\% $=3,56-4,60)$, sendo que, neste grupo, $58,2 \%(n=110)$ apresentaram atividade de cárie.

O CPOD foi estratificado de acordo com o percentual dos seus componentes, segundo como pode ser visualizado no Gráfico 1. N este gráfico, pode-se observar que a maior parte do índice é composto pelo componentecariado, seguido pelo componente restaurado.

A quedana experiência decárieno município delndaiatuba, desdeo ano de 1992, expressa através do índiceCPOD em diversos pontos do tempo, pode ser visualizada no Gráfico 2, bem como os componentes do índice. $O$ valor do CPOD encontrado no ano 2000 não diferiu do encontrado em $2004(p=0,144)$. Entretanto, pode ser observada uma tendência de redução do índice de cárie (CPOD) aos doze anos de idade no município neste período de doze anos. Em relação

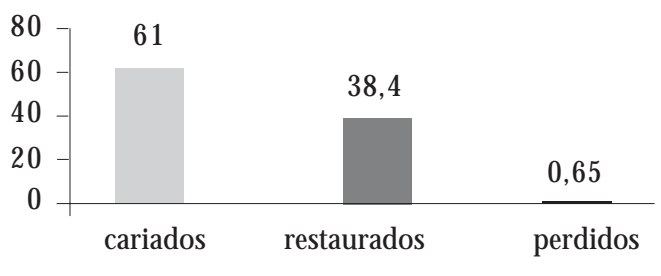

Gráfico 1. Componentes percentuais do índice CPOD em escolares. Indaiatuba (SP), 2004. 
aos seus componentes, verifica-se que, até 0 ano 2000, a maior parte de CPOD era composta pelo componente restaurado; já no ano de 2004, 0 componente mais prevalente foi o cariado.

O Gráfico 3 apresenta a distribuição das fre quências do índiceCPOD nos escolares deI ndaiatuba, no ano de 2004, ou seja, 32\% apresenta-

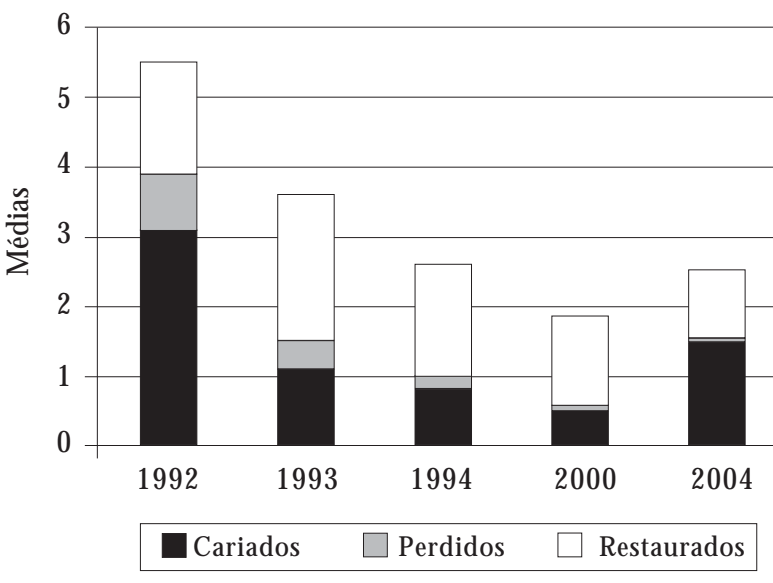

Gráfico 2. Índice CPOD e seus componentes segundo 0 ano de estudo. Indaiatuba (SP), 2004. ram valores deCPOD maiores ou iguaisa 4 . Neste mesmo gráfico, pode-se notar que $38,8 \%$ dos adolescentes examinados eram livres de cárie. Pode-se observar nesta população que um grupo apresenta elevados val ores de CPOD; combinado ao declínio da cárie dentária, observa-se um aumento da desi gualdade na distribuição de cárie dentária, ou seja, maior polarização da doença, pois uma maior proporção de lesões de cárie se concentrou em uma menor proporção de escolares. Observando a curva de Lorenz, pode-se notar que o coeficiente de Gini obtido no presenteestudo foi de 0,602; quanto mais próximo de 10 valor encontrado, maior a desigualdade na distribuição de cárie em uma população, ou seja, enquanto muitas crianças apresentam-selivres de cárie, ainda existem crianças que apresentam elevados valores da doença.

Com a finalidade de interpretar os dados através de outra medida, calculou-seo índiceSiC. O valor do índice SiC foi de 5,97 (IC95\% =5,326,62 ). N os indivíduos pertencentes ao grupo $\mathrm{SiC}$, isto é, que apresentaram índices mais elevados de experiência de cárie $(n=99), 74,7 \% \quad(n=74)$ apresentaram atividade de cárie. Ainda nestegrupo, pode-se verificar que concentrou cerca de $85 \%$ das necessidades de tratamento. N os demaisindivíduos com menor experiência decárie, a necessidade de tratamento restaurador, pulpar e/ou cirúrgico foi deapenas $25 \%$ do total dedentes indicados para tratamento.
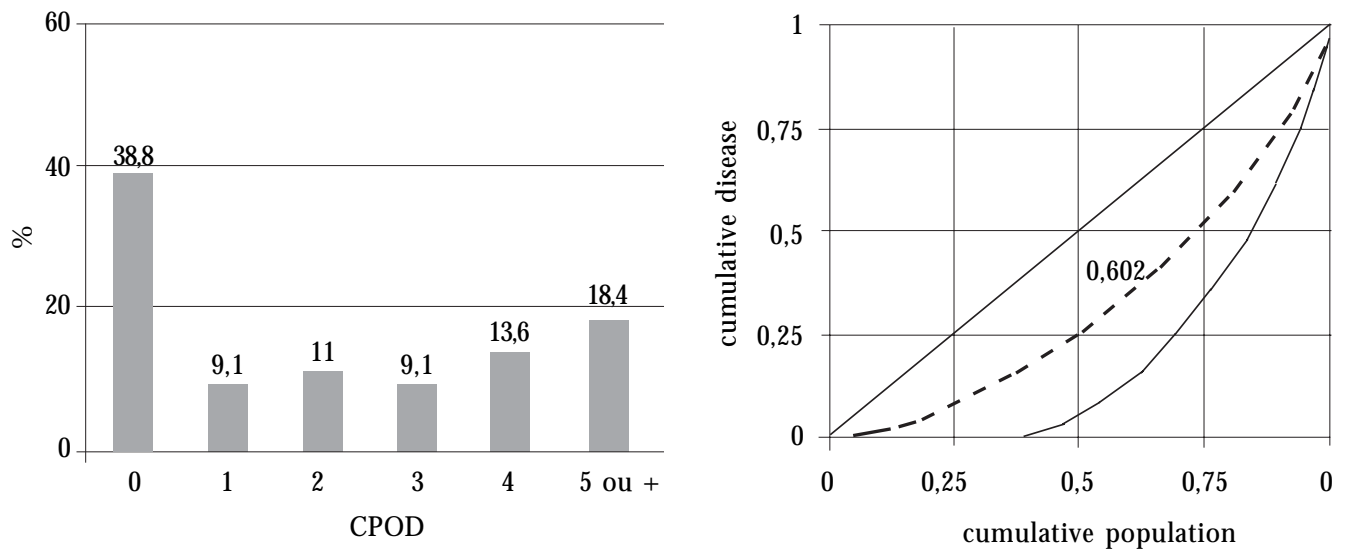

Gráfico 3. Distribuição da frequência e curva de Lorenz para o CPOD de crianças de doze anos. Indaiatuba (SP), 2004. 
No Gráfico 4, podem ser observadas as ne cessidades de tratamento odontológico tanto das crianças com os menores índices de cárie (dois terços dos adolescentes que apresentavam os menores valores do (POD), definidos nesteestudo como grupo Não SiC, e as necessidades nas crianças que compuseram o grupo SiC. Observase que, no grupo $\mathrm{SiC}$, houve muito mais indicação de tratamento restaurador que no grupo não $\mathrm{SiC}$, o que reafirma a desigualdade na distribuição da cárie nos adolescentes que compõe o SiC.

\section{Discussão}

Este estudo foi realizado somente em estudantes de escolas públicas, o que talvez possa não corresponder de forma fidedigna à realidade do município como um todo, restringindo a inferência a esta população. Esta mesma ressalva é pertinente ao grupo de polarização identificado nos resultados.

Analisando-se municípios do Estado de São Paulo que possuem água fluoretada, verificouse, em 1998, para a região de Sorocaba ${ }^{17}$, que 0

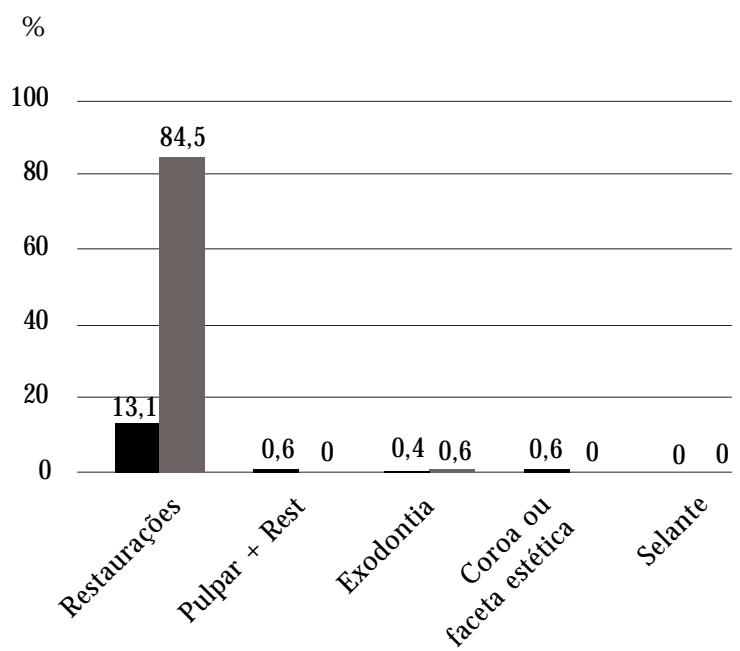

Grupo Não SiC (2/3 com escores mais baixos de CPOD) Grupo SiC (1/3 com escores mais elevados de CPOD

Gráfico 4. Percentual de necessidade de tratamento segundo o nível de experiência de cárie. Indaiatuba (SP), 2004.
CPOD $(2,5)$ foi o mesmo encontrado em 2004 em Indaiatuba. Entretanto, em levantamento realizado no Estado de São Paulo no ano de 2002, a média CPOD foi de 2,52, e quando neste trabaIho os municípios foram divididos segundo a condição defluoretação da água, verificou-seque os municípios com água fluoretada apresentaram CPOD de 2,34, e quando foram considerados apenas os municípios sem água fluoretada, o CPOD foi de 3,51. Assim, nos locais sem fluoretação, esteíndice foi $50 \%$ mais el evado 5 . Internacionalmente, em revisão de literatura na qual são relatados trabal hos realizados na Europa, foram verificados valores inferiores ao encontrado em Indaiatuba ${ }^{18}$.

Neste mesmo contexto, observou-se que 0 componente cariado correspondeu à maioria do índice CPOD (Gráfico 1). Isto pode ser devido ao fato de a amostra ser composta por crianças deescolas públicas. Em estudo realizado na Bahia, verificou-se que estudantes de escolas públicas apresentaram maior componente cariado, enquanto os de escolas particulares, maior componenterestaurado ${ }^{19}$. Além disto, apenas $38,4 \%$ dos dentes com experiência de cárie encontram-se restaurados, refletindo indiretamente uma dificuldade de acesso desta população às ações assistenciais em saúde bucal.

O município de Indaiatuba possui um histórico de levantamentos epidemiológicos em relação à cárie dentária. Deve-se, entretanto, analisar estes dados de forma cuidadosa, pois nos estudos anteriores ao ano de 2000 realizados em Indaiatuba, o critério de diagnóstico da cárie dentária envolvia o exame tátil-visual para o diagnóstico de cárie, com o uso de espelho bucal e sonda exploradora pontiaguda ${ }^{13}$. M esmo com estas ressalvas, é possível observar uma tendência de declínio na experiência de cárie, na ordem de 54\% no período entre 1992 e 2004 (Gráfico 2).

Esta redução na experiência de cárie, visualizada no Gráfico 2, muito provavelmente está relacionada à incorporação de flúor à água (queno município deu-se em 1979) e aos dentifrícios a partir de $1988^{20}$. Esta associação entreuso de produtosfluoretadosediminuição dosíndices decárie no Brasil ébem descrita em uma revisão da literatura realizada por Cury et al. ${ }^{21}$. N este estudo, os autores atribuem a redução nos índices de cáriea principalmentetrês fatores: água fluoretada, dentifrícios fluoretados e programas odontológicos preventivos realizados em escolas.

Ainda no mesmo gráfico, observa-se que, a partir de 1994, O CPOD se estabilizou, mostrando, possivelmente, apenas uma pequena redu- 
ção numérica, num período de dez anos, suge rindo que são necessárias medidas adicionais para reduzir a severidade da cárieem grupos mais específicos da população, conforme será discutido posteriormente.

Quando os indivíduos livres de cárie $(C P O D=0)$ foram excluídos da amostra, o valor do CPOD aumentou consideravelmente, sugerindo-seque, em populações nas quais o percentual de escolareslivres de cárieé elevado, deve-se analisar a média CPOD na população com experiência de cárie, para que a mensuração das ne cessidades de cuidados em saúde seja melhor dimensionada.

A maioria das necessidades de tratamento foram restaurações simples, seguidas de restaurações de duas superfícies (Gráfico 4); entretanto, apesar de $36,6 \%$ dos indivíduos examinados apresentarem atividade de cárie, não houve nenhum caso de indicação de selantes oclusais para estes escolares. Conforme descrito na metodologia, a indicação de selantes para este estudo seguiu as regras de decisão sugerida pela SES/ $\mathrm{SP}^{14}$, mas acredita-se que esta indicação esteja subestimada pelas dificuldades em se detectar os estágios iniciais da doença cárie em estudos desta natureza, principalmente em dentes posteriores. Assim, recomenda-se que estudos mais acurados sejam realizados para dimensionar a real indicação de selantes em saúde pública, como um método complementar para reduzir a incidência da doença cárie em populações com diferentes níveis de experiência de cárie. Um ponto bastante importante no Gráfico 4 foi a alta porcentagem de pessoas com necessidade de restaurações no grupo $\mathrm{SiC}$, o queevidencia a desigualdadena distribuição da cárie nesta população.

Outro dado relevante para a saúde bucal desta população refere-se ao percentual de escolares livres de cárie (38,8\%); quando comparado com municípios na mesma região do estado, quetambém possuem água fluoretada, como Leme $(43,6 \%)^{21}$, Rio Claro $(54,6 \%)^{4}$, Paulínia $(46,1 \%)^{1}$, o município apresentou valores inferiores de indivíduos livres de cárie, denotando condições de saúde bucal menos favoráveis. No Estado de São Paulo, nos municípios com fluoretação, o percentual de livres de cárie foi de $31,8 \%$, em $2002^{5}$, um pouco abaixo do percentual observado no presente estudo.

No Gráfico 3, verifica-se que, apesar do percentual de escolaresquese apresentavam livres de cárieno momento do exameser baixo, verifica-se uma polarização da cárienesta população, ou seja, aproximadamente $20 \%$ das pessoas apresentam valores de CPOD maiores que 4 , denotando que em Indaiatuba não é verificada uma distribuição normal da doença, aos doze anos de idade, o que caracteriza um processo de polarização da cárie. Desta forma, sugere-se que outras medidas, além do índice CPOD, sejam instituídas. A partir desta observação, as autoras realizaram o cálculo do coeficiente deGini, utilizado para a verificação de desigualdades na experiência de cárie.

Devido a esta desigualdade na distribuição de cáries no município verificada através do coeficiente de Gini, foi realizado o cálculo do SiC, que avalia o CPOD em um terço da população com os maiores valores de CPOD. $O$ índice SiC observado nesta amostra foi de 5,97. A OMS propõe como meta para o ano 2015 um SiC menor que 3 aos doze anos de idade ${ }^{22}$. Esteíndice representa 0 CPOD de um terço da população, que apresentou maiores médias CPOD, que pode ser considerado como grupo de risco ou de alta experiência de cárie. Em populações em que existe uma visível polarização da cárie, justifica-se a utilização de medidas de avaliação como o SiC e/ou verificação da presença de manchas brancas ativas na população. Através destas medidas, este grupo pode ser identificado e estratégias de atenção diferenciadas podem ser incorporadas, sendo que resultados satisfatórios têm sido encontrados quando o grupo pertencente ao $\mathrm{SiC}$ recebe cuidados direcionados ${ }^{3}$. Conforme o esperado, a atividade de cárie neste grupo específico foi alta $(74,7 \%)$ e provavelmente estes escolares necessitam de uma atenção em saúde bucal diferenciada e compatível com as necessidades que, conforme descrito nos resultados, consiste de procedimentos de menor custo e pouco complexos. Caso isto não seja implementado, após al gum tempo, esta população com al tos níveis de doença não estará apresentando mais dentes cariados, agravandose a severidade sob controle, agravando-se a severidade de doença e aumentan do a complexidade da intervenção. Portanto, o sistema de saúde deveria direcionar ações no sentido de identificar este grupo da população com níveis mais elevados de doença, ou seja, que apresenta polarização da doença, devendo lançar mão de medidas combinadas e complementares para reduzir globalmenteos indicadores da cáriena população como um todo (estratégias populacionais), com estratégias para grupos de risco em segmentos específicos da população para diminuir as desigualdades na distribuição da doença cárie.

0 estudo periódico do comportamento das princi pais doen ças bucais éimportante, uma vez que é através deste que os municípios mantêm 
um adequado acompanhamento do estado de saúde bucal, podendo, desta forma planejar e avaliar as ações direcionadas a esta população. No caso dos escolares de I ndai atuba, os esforços deveriam estar visando às metas para a saúde bucal da OM S para 2010, uma vez que as para 0 ano 2000 foram atingidas. Além disto, como a cárie dentária de uma forma geral se encontra controlada nesta população, estratégias como 0 coeficientedeGini ea identificação do grupo que compõe o SiC poderiam ser utilizadas como auxiliares na identificação dos grupos mais vulneráveis nesta população, enfatizando-se 0 exame clínico das manchas brancas ativas, nos quais um cuidado diferenciado poderia estar sendo direcionado visando à redução dosíndices de cárie também nestegrupo. Recomenda-secuidado diferenciado aos escolares com níveis mais elevados de cárie dentária (grupo SiC).

Desta forma, pode-se concluir que os escolares do município de Indaiatuba, em 2004, apresentaram baixa experiência de cárie; entretanto, não apresentaram suas necessidades atendidas, uma vez quea maior parte do CPOD era formada do componentecariado, aos doze anos deidade. Além disto, verificou-se que as maiores necessidades de tratamento odontológico eram de ações restauradoras. No grupo SiC (que apresentou os maiores índices de cárie), verificou-se que este apresentou-se bastante elevado $(5,97)$, sendo recomendadas ações direcionadas a este grupo específico de adolescentes.

\section{Colaboradores}

LB Rihs organizou o banco de dados e realizou a análise estatística, elaborou os resultados e redigiu o manuscrito; MLR de Sousa contribuiu na calibração da equipe de examinadores, trabalhou na ideal ização do estudo erealizou a revisão final do manuscrito; S Cypriano contribuiu na calibração da equipe de examinadores, elaborou os objetivos, resultados e discussão do manuscrito e revisou a versão final do manuscrito; $N M$ Abdalla participou do planejamento regional do estudo e da revisão final do trabalho. 


\section{Referências}

1. Gomes PR, Costa SC, Cypriano S, Sousa M LR. Paulínia, São Paulo, Brasil: Situação da Cárie dentária com relação às metas OM S 2000 e 2010. Cad Saude Publica 2004; 20(3):866-70.

2. Nadanovsky P, Sheiham A. Relative contribution of dental services to the changes in caries levels of 12year-oldchildren in 18 industrialized countries in the 1970 s and early 1980s. Community Dent Oral Epidemiol 1995; 23:331-339.

3. Pieper K, Schulte AG. The decline in dental caries among 12-year-old children in Germany between 1994 and 2000. Community Dent H ealth 2004; 21:199206.

4. Hoffmann RHS, Cypriano S, Sousa MLR, Wada RS. Experiência de cárie dentária em crianças de escolas públicas e privadas de um município com água fluoretada. Cad Saude Publica 2004; 20:522-528.

5. Secretaria do Estado da Saúde de São Paulo. Universidade de São Paulo. Condições de Saúde Bucal no Estado de São Paulo em 2002. São Paulo: Universidade de São Paulo; 2002.

6. Antunes JLF, Narvai PC, Nugent ZJ. M easuring inequalities in the distribution of dental caries. Community Dent Oral Epidemiol 2004; 32(1):41-48.

7. Antunes JLF, Peres MA, M ello, TRC, Waldman EA Multilevel assessment of determinants of dental caries experience in Brazil. Community Dent Oral Epidemiol 2006; 34(2):146-152.

8. Enjary C, Tubert-Jeannin S, Manevy R, Roger-Leroi $V$, Riordan PJ. Dental status and measures of deprivation in Clermont-Ferrand, France. Community Dent Oral Epidemiol 2006; 34(5):363-371.

9. Marthaler T, Menghini G, Steiner M. Use of Significant Caries Index in quantifying the changes in caries in Switzerland from 1964 to 2000. Community Dent Oral Epidemiol 2005; 33(3):159-166.

10. Nishi M, Stjernswärd J, Carlsson P, Bratthall D. Caries experience of some countries and area expressed by the Significant Caries Index. Community Dent Oral Epidemiol 2002; 30(4):296-301.

11. Brasil. M inistério da Saúde. Projeto SB 2000 - Condições de Saúde Bucal da população brasileira no ano 2000. Manual do examinador. Brasília: Ministério da Saúde; 2001.

12. World Health Organization. Oral health surveys, basic methods. $4^{\text {th }}$ ed. Geneva: WHO; 1997.
13. World Health Organization. Oral health surveys: Basic methods. $3^{\text {rd }}$ ed. Geneva: WHO; 1987.

14. Secretaria de Estado da Saúde de São Paulo. Levantamento das condições de saúde bucal - Estado de São Paulo, 1998: caderno de instruções. São Paulo: Secretaria de Estado da Saúde de São Paulo; 1998.

15. Nyvad B, Machiulskiene V, Baelum V. Reliability of a new caries diagnostic system differentiating between active and inactive caries lesions. Caries Res 1999; 33(4):252-260.

16. Faculdade de Odontologia. Universidade de São PauIo. Gini calculation. [site da Internet] [acessado 2006 jul 19]. Disponível em: http://www.fo.usp.br/arquivos/Gini_calculation_for_caries_distribuition. zip

17. Cypriano S, Pecharki GD, Sousa M LR, Wada RS. A saúde bucal de escolares residentes em locais com ou sem fluoretação nas águas de abastecimento público na região de Sorocaba, São Paulo, Brasil. Cad Saude Publica 2003; 19(4):1063-1071.

18. M arthaler TM. Changes in dental caries 1953-2003. Caries Res 2004; 38(3):173-181.

19. Cangussu MCT, Castellanos RA, Pinheiro MF, Albuquerque SR, Pinho $C$. Cárie dentária em escolares de 12 e 15 anos de escolas públicas e privadas de Salvador, Bahia, Brasil, em 2001. Pesqui O dontol Bras 2002; 16(4):379-384.

20. Cury JA. Dentifrícios fluoretados no mercado brasileiro e seu potencial como método preventivo. Salvador: Associação Brasileira de Odontologia; 1989.

21. Cury JA, Tenuta LMA, Ribeiro CCC, Paes Leme AF. The Importance of Fluoride Dentifrices to the Current Dental Caries Prevalence in Brazil. Braz Dent J 2004; 15(3):167-174.

22. World Health Organization. The World Oral Health Report, 2003. Continuous improvement of oral health in the 21st century - the approach of the WHO Global Oral Health Program. [site da Internet] 2003 [acessado 2007 fev 12]. Disponível em: http://www.who.int/ oral_health/media/en/orh_report03_en.pdf

Artigo apresentado em 26/03/2008

Aprovado em 28/08/2008 